\title{
Three-dimensional ray tracing on Delaunay-based reconstructed surfaces
}

\author{
Sergio Ortiz, ${ }^{1, *}$ Damian Siedlecki, ${ }^{1,2}$ Laura Remon, ${ }^{1}$ and Susana Marcos ${ }^{1}$ \\ 'Instituto de Óptica "Daza de Valdés", Consejo Superior de Investigaciones Científicas, \\ C/Serrano 121, 28006 Madrid, Spain \\ ${ }^{2}$ Institute of Physics, Wroclaw University of Technology, \\ Wybrzeze Wyspianskiego 27, 50370 Wroclaw, Poland \\ *Corresponding author: sortiz@io.cfmac.csic.es \\ Received 27 May 2009; accepted 13 June 2009; \\ posted 19 June 2009 (Doc. ID 112019); published 1 July 2009
}

\begin{abstract}
A method of ray tracing for free-form optical surfaces has been developed. The ray tracing through such surfaces is based on Delaunay triangulation of the discrete data of the surface and is related to finite-element modeling. Some numerical examples of applications to analytical, noisy, and experimental free-form surfaces (in particular, a corneal topography map) are presented. Ray-tracing results (i.e., spot diagram root-mean-square error) with the new method are in agreement with those obtained using a modal fitting of the surface, for sampling densities higher than $40 \times 40$ elements. The method competes in flexibility, simplicity, and computing times with standard methods for surface fitting and ray tracing. (C) 2009 Optical Society of America
\end{abstract}

OCIS codes: $\quad 080.1753,080.2720,330.7326,330.4875$.

\section{Introduction}

A large variety of ray-tracing algorithms can be observed in the literature. Many of them involve ray tracing through nonhomogeneous or gradient index media [1-4]. Some others describe the light propagation in birefrigent materials [5-8] or optical fibers (waveguides) $[9,10]$. However, except for a few papers [11-14], not many works in the literature explain a precise and efficient way of finding the intersection points of rays with optical surfaces. Although this problem seems to be relatively trivial, our experience in physiological optics shows that it is not, particularly if "noisy" or free-form surfaces are of interest. Interesting examples of such surfaces are surfaces of biological origin, for example, the cornea of the eye. The anterior corneal surface plays a very important role in focusing light on the retina. Its topography has a major influence on the optical performance

0003-6935/09/203886-08\$15.00/0

(C) 2009 Optical Society of America of the eye, including higher-order aberrations [15-24].

This paper presents a three-dimensional (3D) technique of tracing rays through optical surfaces of any type. The method is based on the Delaunay triangulation $[25,26]$, which is a well-known method in finite-element modeling and graphical ray tracing that is used for rendering photorealistic images [27-29]. To the best of our knowledge, this method has never been implemented in geometrical optics. Typically, optical analysis software, such as ZEMAX [30] uses a least-mean-square method together with linear or spline interpolation of the discrete surface points. The least-mean-square method is relatively fast and its accuracy, even for analytical, surfaces is acceptable. However, it depends strongly on the sampling density of the surface. The Delaunay method proposed in the current study can optimize computations of optical analysis of optical systems, regarding particularly the intersection points of a ray with an optical surface.

This finite-element technique is an approach that was developed in our laboratory for the purposes of 
the optical distortion correction [31] of 3D optical coherence tomography (OCT) images $[32,33]$, where the real shape of the surface is not known $a$ priori and has to be estimated.

\section{Delaunay Decomposition}

Delaunay decomposition is a well-described $[25,26]$ mathematical method used in computational geometry. It uses the property of the convex hull (envelope) of the discrete set of points $\{X, Y\}$. By means of the Delaunay triangulation method, one can approximate any surface with a set of flat triangles, as is usually done in the finite-element method (FEM) [34-38] (Fig. 1.). The number of obtained triangles is related to the number of points in the grid and the number of vertices on the convex hull. If $m$ is the number of points in the grid, and $l$ is the number of vertices, then the number of triangles $t$ is defined by the following equation:

$$
t=2 m-2-l .
$$

Because all the triangles are flat, one can easily calculate the vector normal to each of them and use it further in the Snell's law accomplishment. This makes the Delaunay triangulation method very convenient and relatively easy to implement to optical ray tracing.

\section{A. Description of the Algorithm}

In a first step, it is necessary to arrange adequately the data of the surface. The surface should be described by the set of points $\{X, Y, Z\}$ corresponding to a discrete sample of the surface topography. If the surface is analytical, the set $\{X, Y, Z\}$ can be generated as a mesh with regular sampling. The optical surface can be the result of a topographical measurement performed on an irregular or even random sampling pattern. On a set of $\{X, Y\}$, one can perform two-dimensional Delaunay decomposition, where each point from the discrete data set $\{X, Y\}$ is one

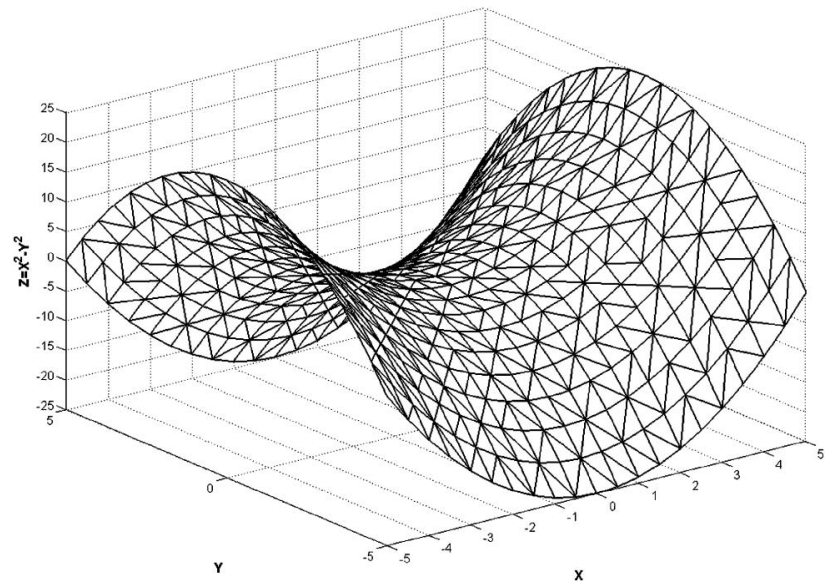

Fig. 1. Function $z(x, y)=x^{2}-y^{2}$ represented as a set of triangles to illustrate the Delaunay triangulation. The discrete data of the function are situated in vertices of triangles. The axes are given in arbitrary units. of the vertices of at least two triangles. This results in a $3 \mathrm{D}$ approximation of the surface $Z=f(X, Y)$.

The next step is to find one particular triangle, which is hit by a ray denoted by its initial position, $R_{0}$, and an optical vector at this point, $\overline{K_{0}}$ :

$$
R_{0}=\left(\begin{array}{l}
x_{0} \\
y_{0} \\
z_{0}
\end{array}\right), \quad \overline{K_{0}}=\left[\begin{array}{c}
k_{x 0} \\
k_{y 0} \\
k_{z 0}
\end{array}\right],
$$

where $k_{x 0}, k_{y 0}$, and $k_{z 0}$ represent directional cosines.

One can test each triangle separately, although this implies a relatively large computational effort, especially if the surface is large and the grid $\{X, Y\}$ is dense. Alternatively, a much more efficient way to do it is to assess the region of interest $a$ priori by finding the minimum Euclidean distance between the straight line given by the optical vector

$$
R=R_{0}+t \overline{K_{0}},
$$

(with $t$ as the length of vector $\overline{K_{0}}$ and a real number) and each of the points from the set $\{X, Y, Z\}$. This distance is given by the following formula:

$$
d_{i}=\left|\overline{w_{i}}-\left(\overline{w_{i}} \cdot \overline{K_{0}}\right) \overline{K_{0}}\right|
$$

where $\overline{w_{i}}$ is a vector obtained by subtracting the coordinates of the point $R_{0}$ from the coordinates of the $i$ th point from the set $\{X, Y, Z\} \quad\left(\left\{X_{i}, Y_{i}, Z_{i}\right\} \in\right.$ $\{X, Y, Z\})$. If $d_{m}=\min \left(d_{i}\right)$ is found, this means that the ray hits one of the triangles surrounding the vertex $P_{m}=\left(X_{m}, Y_{m}, Z_{m}\right)$. Following this procedure, instead of testing thousands or even millions of triangles [Eq. (1)], one can limit the area of interest to (on average) six triangles (Fig. 2). In the case of a random sampling pattern, the number of triangles depends on the configuration of neighbors. The average number of triangles surrounding each vertex is characteristic of the Delaunay triangulation [25,26]. With the number of possible triangles significantly reduced, one can easily find the common point to

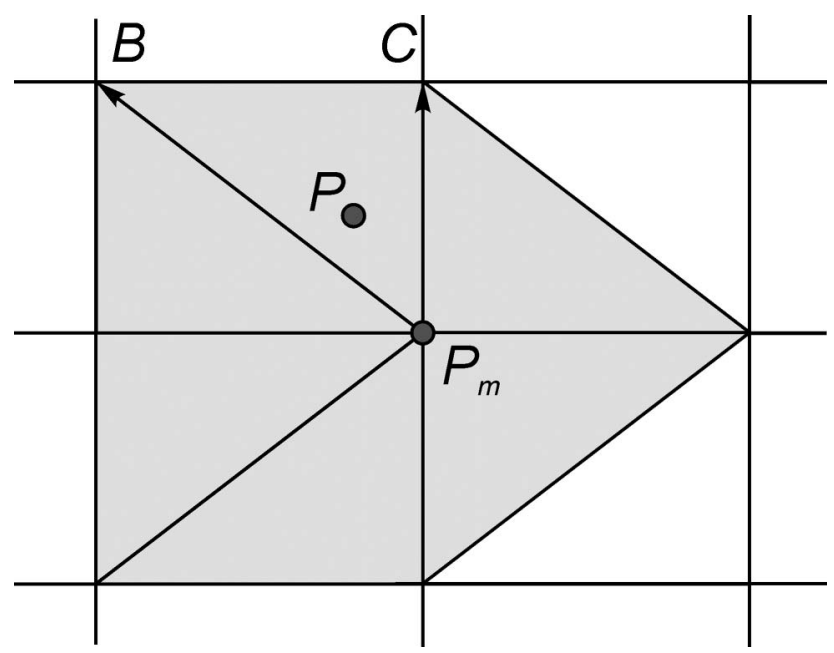

Fig. 2. Graphical illustration of a point $P_{m}$ that is a common vertex of six neighboring triangles and of the barycentric technique. 
the ray given by Eq. (3), and each of the triangles of interest. This allows obtaining (on average) six different points on six different planes defined by six different triangles.

Additional calculations are required to obtain the exact triangle that is intersected by a ray. The proper facet can be found by means of the barycentric technique [39], which is very commonly used together with Delaunay triangulation. Let us consider a triangle given by points $P_{m}, B$, and $C$, with $P_{m}$ as a reference point (Fig. 2). Each point of the plane containing the triangle $P_{m}^{-} B C$ can be described as a linear combination of two vectors defining the plane:

$$
P=P_{m}+u \cdot \overline{P_{m} B}+v \cdot \overline{P_{m} C},
$$

where $\{u, v\} \in \mathbb{R}$. If we have the coordinates of the point $P$, after some simple mathematical operations one can get the following formulas for $u$ and $v$ :

$$
\begin{aligned}
u & =\frac{\left(\overline{P_{m} B} \cdot \overline{P_{m} B}\right)\left(\overline{P_{m} P} \cdot \overline{P_{m} C}\right)-\left(\overline{P_{m} B} \cdot \overline{P_{m} C}\right)\left(\overline{P_{m} P} \cdot \overline{P_{m} B}\right)}{\left(\overline{P_{m} C} \cdot \overline{P_{m} C}\right)\left(\overline{P_{m} B} \cdot \overline{P_{m} B}\right)-\left(\overline{P_{m} C} \cdot \overline{P_{m} B}\right)\left(\overline{P_{m} B} \cdot \overline{P_{m} C}\right)}, \\
v & =\frac{\left(\overline{P_{m} C} \cdot \overline{P_{m} C}\right)\left(\overline{P_{m} P} \cdot \overline{P_{m} B}\right)-\left(\overline{P_{m} C} \cdot \overline{P_{m} B}\right)\left(\overline{P_{m} P} \cdot \overline{P_{m} C}\right)}{\left(\overline{P_{m} C} \cdot \overline{P_{m} C}\right)\left(\overline{P_{m} B} \cdot \overline{P_{m} B}\right)-\left(\overline{P_{m} C} \cdot \overline{P_{m} B}\right)\left(\overline{P_{m} B} \cdot \overline{P_{m} C}\right)},
\end{aligned}
$$

From the values of $u$ and $v$ one can assess if a particular point $P$ belongs to the triangle or not. If $u$ or $v$ is $<0, u$ or $v>1$, and $u+v>1$, the point $P$ is out of the triangle. Alternatively, if conditions $0 \leq u+v \leq 1$, $0 \leq u \leq 1$, and $0 \leq v \leq 1$ are met, this means that the point $P$ belongs to the particular triangle [40].

Once the triangles of the surface where rays intersect the surface have been determined, the $z$ value can be found by means of linear interpolation, as the intersection of three planes, or alternatively, using more complex methods of interpolation, such as the values of the neighbors (B-splines) or radial base functions [41]. The use of radial base functions does not require the use of a regular grid of sampling and potentially could allow reducing the density of the sampling (and computational time) without compromising accuracy. In this study, we have implemented several of these functions: cubic, multiquadric [42], and thin plate spline [43], besides the simplest linear interpolation. The algorithm [44] consists of fitting a number of coefficients using the values of $x, y$, and $z$ of control points called nodes. The control points in our case are the points belonging to the triangle within the ray impacts. Once the coefficients are obtained, the value of the $z$ coordinate can be easily obtained simply by substitution of the value of the $x$ and $y$ coordinates into the following equation:

$$
f(x, y)=c_{0}+c_{1} x+c_{2} y+\sum_{i=1}^{n} c_{i+2} \varphi\left(d_{i}\right)
$$

where $c$ denotes the coefficients of the interpolation, $\varphi$ is a radial base function (cubic, multiquadric, thin plate, etc.), $d_{i}$ is the distance between the $i$ th node and the point to be interpolated, and $n$ is the number of nodes. Further details can be found in the literature [44].

For the linearly interpolated method, the normal to the surface at point $P$ is associated to the characteristic vector of the facet to which this point belongs, with the following equations:

$$
\hat{N}=\frac{\overline{P_{m} B} \times \overline{P_{m} C}}{\left|\overline{P_{m} B} \times \overline{P_{m} C}\right|} \quad \text { or } \quad \hat{N}=\frac{\overline{P_{m} C} \times \overline{P_{m} B}}{\left|\overline{P_{m} C} \times \overline{P_{m} B}\right|},
$$

for a convex or concave initial surface.

In the case of a more complex method of interpolation, the normals can be assessed using the partial derivatives with respect to $x$ and $y$ and, finally, performing the cross product.

Once we have a point $P$ that belongs to the surface and a normal of the surface at this point, $\hat{N}$, any of the 3D implementations of the Snell's law can be applied, i.e., the formula described by Sharma et al. [2]:

$$
n^{\prime} \overline{K_{1}}=n \overline{K_{0}}+w \hat{N}
$$

where $\overline{K_{1}}$ is the optical vector after refraction, $n$ and $n^{\prime}$ are refractive indices before and after the surface, respectively, and $w$ is calculated as follows [45]:

$$
w=\sqrt{n^{2}-n^{\prime 2}+v^{2}}-v,
$$

where $v=\overline{K_{0}} \cdot \hat{N}$.

In Section 3 we present some numerical examples of application of the developed method to various surfaces that can be encountered in optical design and physiological optics. The three types of surfaces that we describe are analytical surface, noisy surface, and free-form surface, in particular, a corneal topography from a normal subject obtained from a Placido disc corneal videokeratopographer (Atlas, Zeiss). For this example, a semianalytical algorithm of a raytracing algorithm through a surface approximated by a set of Zernike polynomials was developed and programmed in MATLAB for the purposes of comparison. The free-form surface data under consideration was approximated by 36 Zernike polynomials $[46,47]$ with a $3 \mathrm{~mm}$ normalization radius, using a standard least-mean-square method. The intersection points of ray paths with the approximated surface were found by means of a least-mean-square method (because of the relative complexity of the expansion formula), while the normals to the surface at these points were calculated analytically by the partial derivatives. We refer to this algorithm as "semianalytical."

\section{Numerical Examples}

To demonstrate the possibilities and accuracy of the algorithm described in Section 2, we will present examples of $3 \mathrm{D}$ ray tracing using the described methods on several types of surfaces (noise-free 


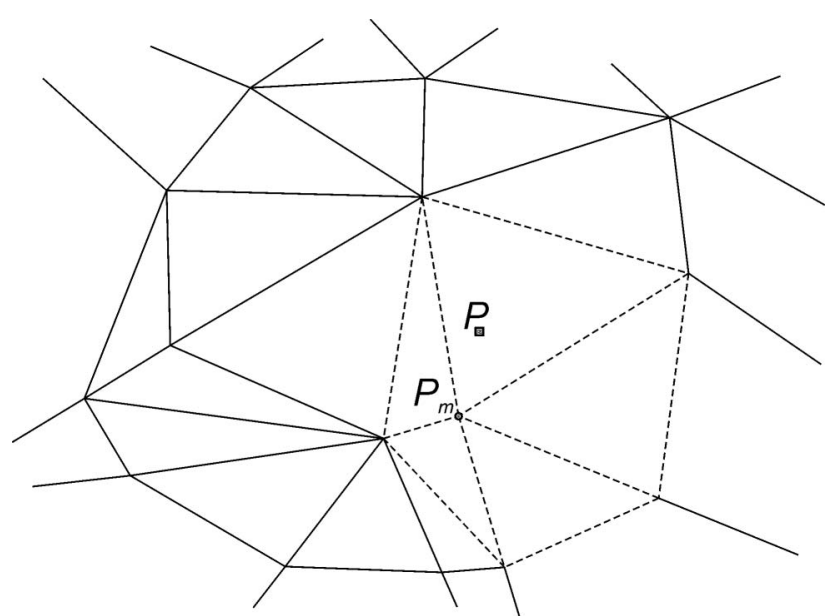

Fig. 3. Example of a random grid on a plane. The dashed lines form triangles surrounding point $P_{m}$.

and noisy analytical surface, and free-form experimental surface).

\section{A. Analytical Surface With a Regular and Random Grid}

A conic surface has been considered for comparative purposes of ray tracing on the analytical and Delaunay decomposed surface. In particular, the first surface of an achromatic lens (AC508-100-B) from the Thorlabs optical elements catalog has been studied. The radius of the anterior surface of this lens is $65.77 \mathrm{~mm}$, and the conic constant $k=1$ (according to Malacara's notation [48]). The refractive index of the

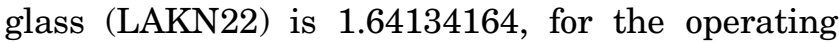
wavelength of $855 \mathrm{~nm}$. In order to test the accuracy of the method proposed, two different outcomes were evaluated (distance between the intersection points and refracted angle difference) from the ray tracing on the analytical (using Zemax, a commercial optical design and analysis software) and Delaunay decomposed surface with use of linear interpolation and with various sampling densities (programmed in MATLAB, Mathworks, Nantick, Massachusetts) for 200 rays entering the lens aperture at different heights. Our simulations showed that the average difference in distance estimated from the two methods ranged from 0.005 to $0.150 \mu \mathrm{m}$ and the average difference in angles ranged from 0.005 to 1.79 arc min. For sufficiently high density sampling grids, these values are slightly larger than the Raleigh criterion ( 0.07 arc min, for the dimensions of the tested lens).

The same accuracy test has been performed for an analytical surface but defined on a random grid (Fig. 3). The difference in distances between the two methods varied from 0.004 to $0.155 \mu \mathrm{m}$, and in angles from 0.17 to 1.94 arc min.

\section{B. Noisy Surface With a Regular Grid}

The next step is to explore the possibilities of the technique on noisy surfaces, for which random noise of Gaussian spectrum has been added to the analytical topography of the surface. The rms of the height deviations has been kept below $\lambda / 8$, and generated using the algorithm provided by Tsang [49], with correlation lengths of 1 and $1000 \mu \mathrm{m}$ (which meet the Beckmann [50] criterion for rough surfaces). The onedimensiona Tsang method has been extended to the two-dimensional surfaces assuming circular symmetry (Fig. 4), and repeated ten times for six different sampling densities ranging from 100 to 2500 points per square millimeter. The simulated surface would be consistent to that produced by a lathe or optical driller.

Table 1 shows the results from these simulations for linearly interpolated data points. Difference values range from 0.021 to $0.204 \mu \mathrm{m}$ and angles from 0.23 to 3.67 arc $\min$.

\section{Free-Form Surface (Corneal Topography)}

Free-form surfaces present a real challenge for optical design and analysis, as their surfaces cannot be easily described analytically. Examples of such surfaces include those of progressive addition lenses (PALs) designed to produce a power gradient across the lens to compensate for the accommodative loss in presbyopia $[51,52]$. Another example of a free-form optical surface can be the topography of the human cornea. Currently it is possible to measure the

(a)
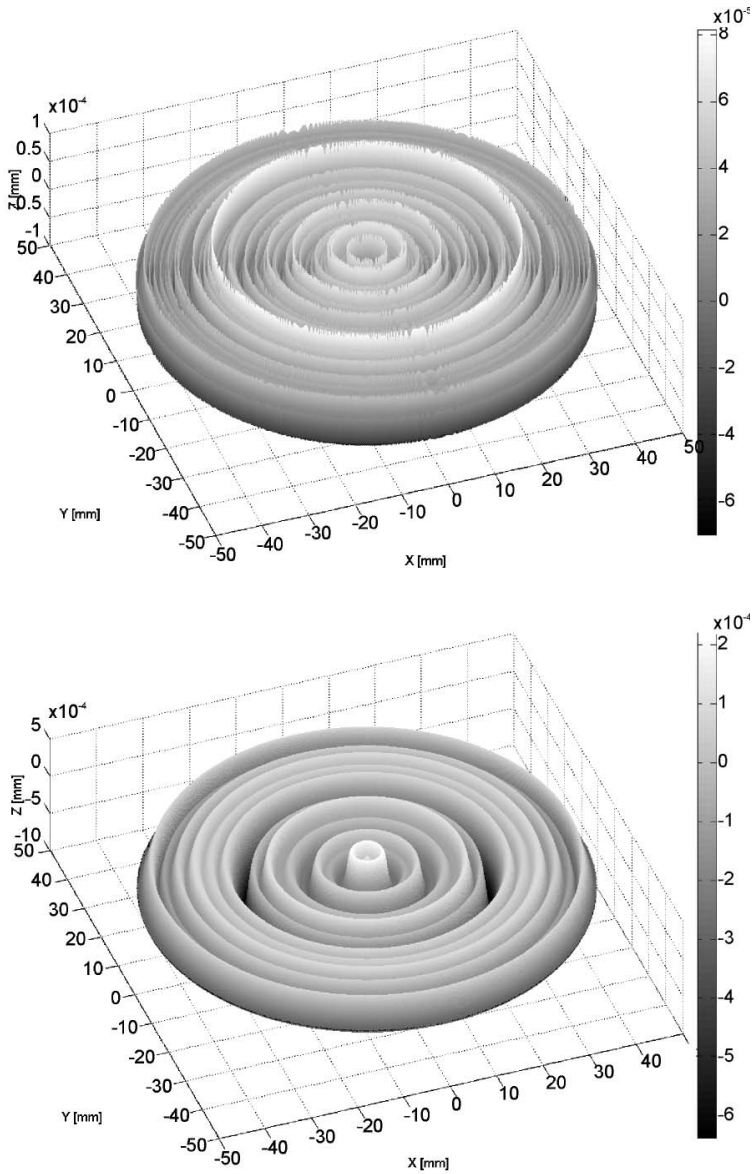

(b)

Fig. 4. Noise generated for simulations of realistic noisy surfaces: (a) $\mathrm{rms}=855 / 8 \mathrm{~nm}$ and correlation length equal to $1 \mu \mathrm{m}$ and (b) $\mathrm{rms}=855 / 8 \mathrm{~nm}$ and correlation length equal to $1000 \mu \mathrm{m}$. 
Table 1. Accuracy of the Algorithm for Different Sampling Grid Densities (Noisy Surface) ${ }^{a}$

\begin{tabular}{|c|c|c|c|c|c|c|c|c|}
\hline \multirow[b]{3}{*}{$\begin{array}{c}\text { Sampling grid } \\
\text { density }\left(1 / \mathrm{mm}^{2}\right)\end{array}$} & \multicolumn{4}{|c|}{ Coherence Length $1 \mu \mathrm{m}$} & \multicolumn{4}{|c|}{ Coherence Length $1000 \mu \mathrm{m}$} \\
\hline & \multicolumn{2}{|c|}{ Distance Difference $(\mu \mathrm{m})$} & \multicolumn{2}{|c|}{ Angle Difference (arc min) } & \multicolumn{2}{|c|}{ Distance Difference $(\mu \mathrm{m})$} & \multicolumn{2}{|c|}{ Angle Difference (arc min) } \\
\hline & Mean & $\begin{array}{l}\text { Standard } \\
\text { deviation }\end{array}$ & Mean & $\begin{array}{l}\text { Standard } \\
\text { deviation }\end{array}$ & Mean & $\begin{array}{l}\text { Standard } \\
\text { deviation }\end{array}$ & Mean & $\begin{array}{l}\text { Standard } \\
\text { deviation }\end{array}$ \\
\hline 100 & 0.156 & 0.016 & 0.749 & 0.109 & 0.204 & 0.154 & 3.673 & 0.305 \\
\hline 400 & 0.045 & 0.023 & 0.233 & 0.132 & 0.192 & 0.140 & 1.942 & 0.287 \\
\hline 900 & 0.020 & 0.018 & 0.598 & 0.344 & 0.135 & 0.094 & 0.153 & 0.135 \\
\hline 1600 & 0.032 & 0.020 & 0.672 & 0.423 & 0.149 & 0.088 & 0.996 & 0.266 \\
\hline 2500 & 0.021 & 0.014 & 1.013 & 0.521 & 0.194 & 0.142 & 0.213 & 0.118 \\
\hline
\end{tabular}

${ }^{a}$ Results for 200 rays along a $25 \mathrm{~mm}$ pupil diameter.

anterior surface elevations of the human cornea with satisfactory accuracy, although an analytical representation is challenging $[18,53,54]$.

Let us consider the raw data from a corneal topography measurement [Fig. 5(a) and 5(b)]. The surface is subject to noise due to experimental errors (camera, tear film, motion) and defined on an irregular grid. Although a common description of the corneal topography is a Zernike polynomial expansion $[46,47,55,56]$, the optimal number of terms that best describes the surface has been debated [57]. The algorithm presented gives the opportunity to trace rays through an optical surface given by raw elevation data points from corneal videokeratopography without any additional approximation. Figure 5(c) shows a ray tracing on a corneal elevation map from a Placido rings corneal videokeratopographer reconstructed using the Delaunay decomposition. If the data are not subject to artefacts (i.e., because of eye lashes) the device is able to provide the elevation data sampled uniformly every $2^{\circ}$ on each of the 24 Placido rings.

For purposes of comparison, the corneal topography from Fig. 5(a) was fitted by a seventh-order polynomial expansion (with 36 terms). The corre- sponding Zernike coefficients and the accuracy of the approximation are presented in Fig. $\underline{6}$. The Zernike expansion was uniformly sampled over the area of interest and then the finite-element raytracing algorithm was applied.

Figure 7(a) compares the results of a ray tracing (200 randomly distributed rays) for various representations of the corneal surface and the ray-tracing algorithms. The values of the rms of the spot diagrams were taken at a distance of $27.72 \mathrm{~mm}$, which is approximately the focal distance of this particular surface, and were used as a metric to compare the outcomes from different fittings (Zernike and Delaunay with different interpolation methods). The first data (labeled as "RawData") correspond to a ray tracing performed directly on a reconstruction of the surface raw elevation data (without prior smoothing) with a Delaunay representation (with the different methods of interpolation, shown with different symbols). The horizontal line corresponds to the Zernike fitting and semianalytical method for ray tracing. The rest of the data represent results from a ray tracing of the same surface fit by a seventh-order Zernike polynomial expansion, followed by Delaunay reconstruction with different

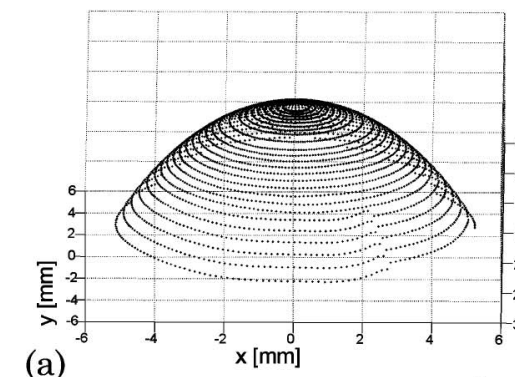

(a)

(b)

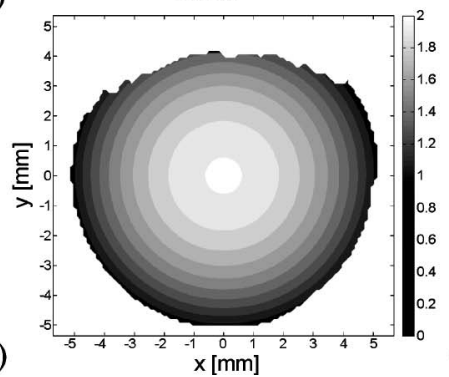

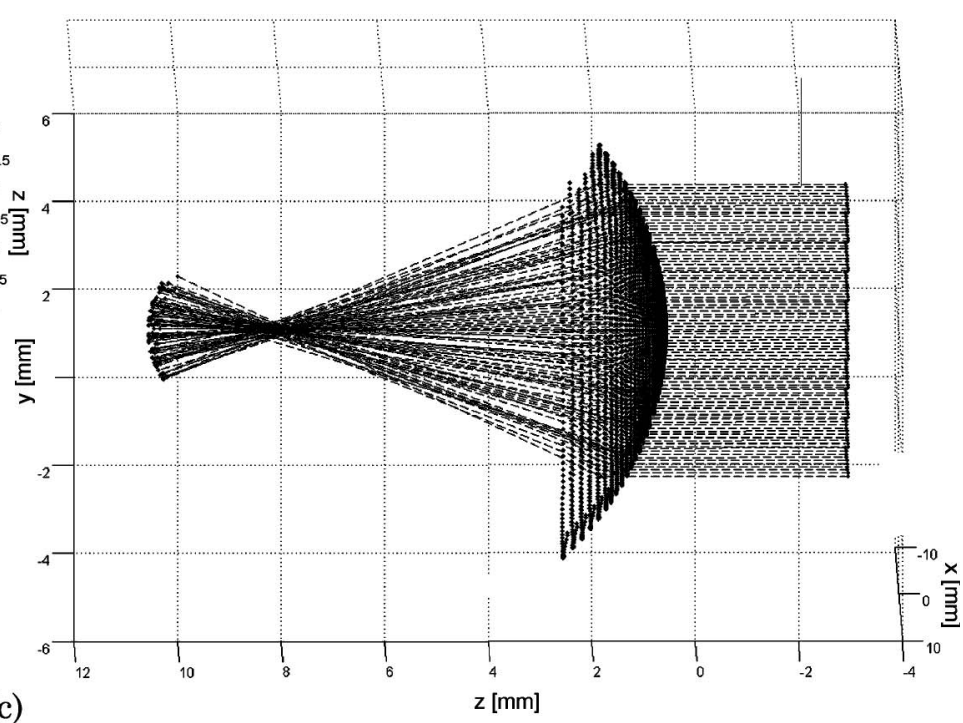

(c)

Fig. 5. Example of a free-form surface: (a) raw (discrete) data from a corneal topography obtained from Placido ring corneal videokeratography, (b) the elevation of the anterior corneal surface, and (c) ray-tracing visualization through the anterior surface of the cornea. 


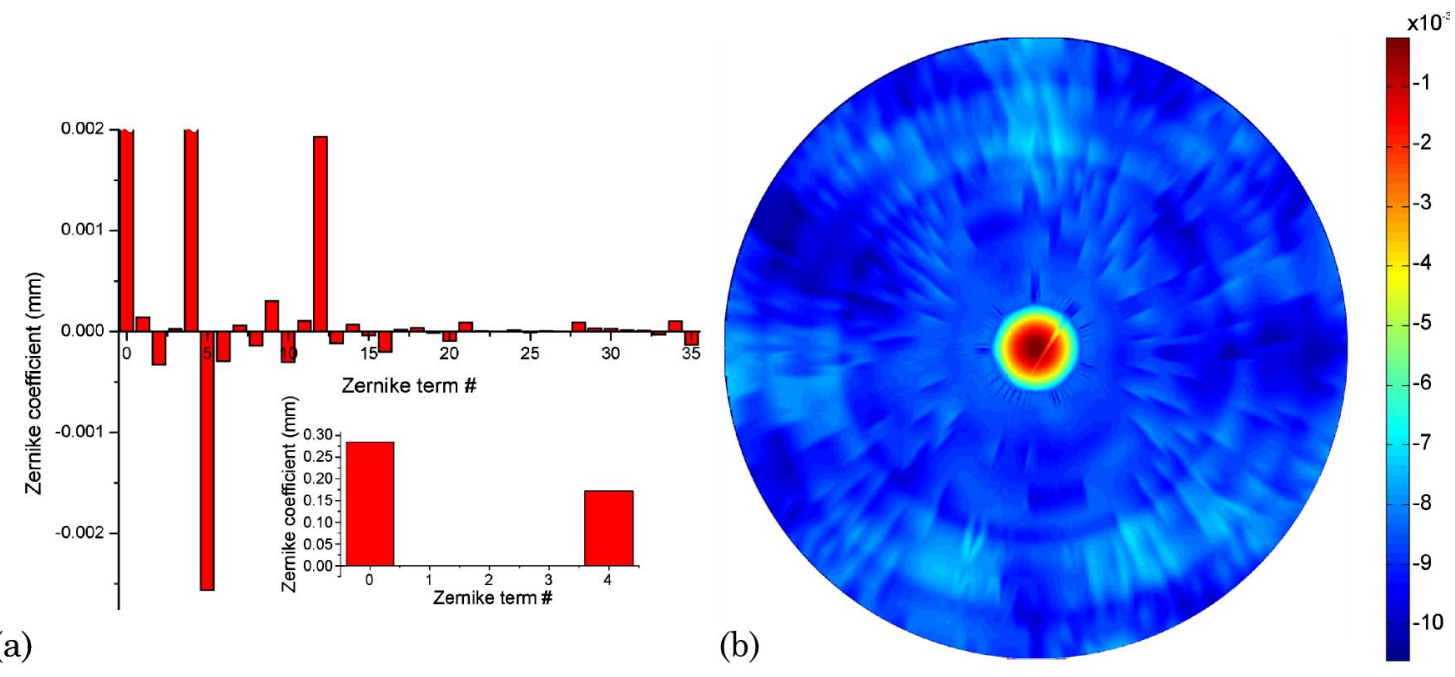

Fig. 6. (Color online) Results of the seventh-order Zernike polynomial expansion of the corneal elevation map of Fig. 5 . (a) Zernike coefficients for a $3 \mathrm{~mm}$ normalization radius. The inset in (a) shows the values of the zeroth and fourth Zernike terms as they are beyond the original scale. (b) Difference between raw data (interpolated with cubic splines) and Zernike polynomial fit. The rms of the difference is $1.4 \mu \mathrm{m}$. The surfaces were set to have the same $Z$ value in the center.

sampling densities (ranging from $7 \times 7$ up to $100 \times$ 100 per $\mathrm{mm}^{-2}$, in the horizontal axis) and different interpolation methods (represented by different symbols). It is remarkable that the values of $\mathrm{rms}$ for finite-element ray tracing for raw data with the use of different interpolation methods is significantly higher than rms for the surface approximated by Zernike polynomials. The Zernike representation smooths the data from the topographical measurement, while the FEM directly uses the raw data and performs the interpolation. The rms values for FEMs on the data approximated by Zernike polynomials converge to the value of $13.6 \mu \mathrm{m}$ achieved by means of the semianalytical method ray tracing with use of Zernike approximation. For cubic and thin plate interpolation methods, the rms values are very similar to the Zernike semianalytical ray tracing even for relatively low sampling densities $(7 \times 7)$, while the linear and multiquadric interpolation methods require denser samplings. Figure 7(b) presents the computational time as a function of sampling density, for 200 rays randomly distributed in the aperture. The finite-element algorithms are competitive in terms of execution time of the ray-tracing procedure for low but sufficient sampling densities. Computational time increases exponentially with sampling density for the Delaunay methods, as expected given the increase of the number of facets.

Figure $\underline{8}$ presents a comparison of the computational time as a function of the number of rays to
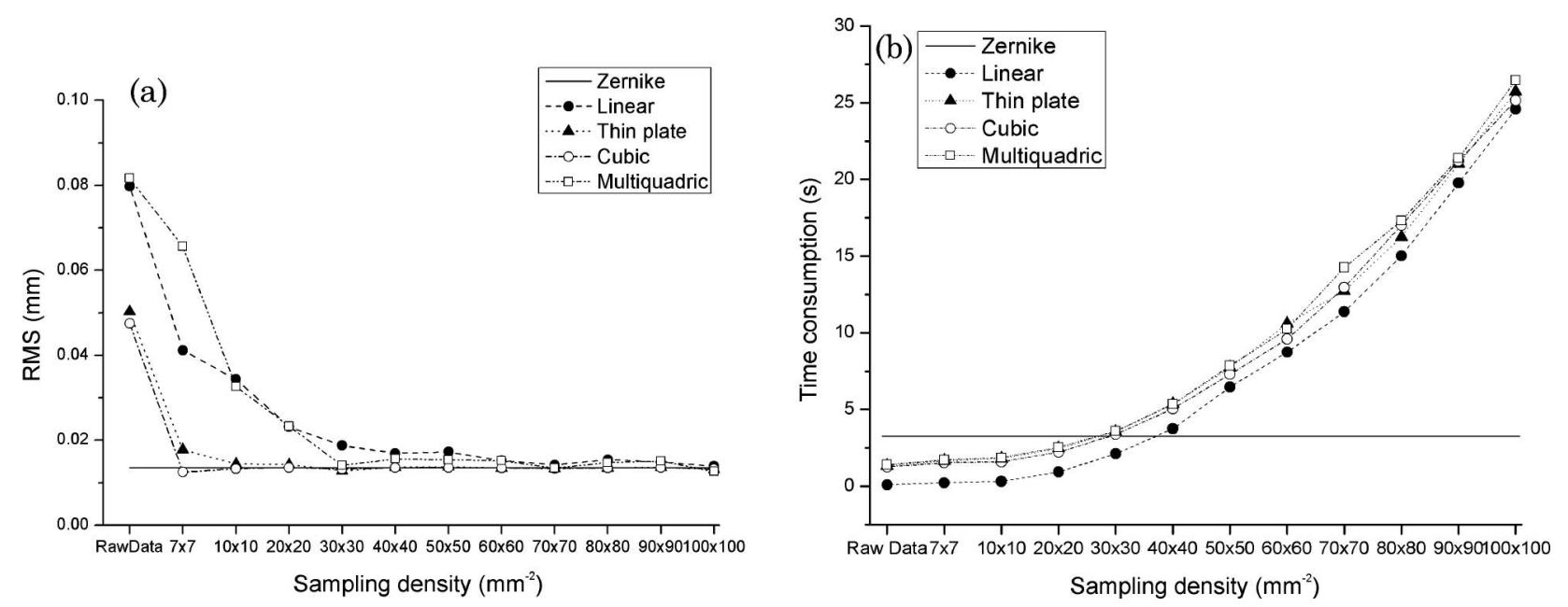

Fig. 7. (a) RMS of the spot diagram taken at the distance $27.72 \mathrm{~mm}$ behind the cornea and (b) computational time, as a function of sampling density for different methods of interpolation in the finite-element ray-tracing algorithm. The position "RawData" in the horizontal axis denotes results from the ray tracing on a direct Delaunay reconstruction from the raw elevation data (with no sampling or Zernike smoothing). For all other cases, the raw data were first approximated by a seventh-order Zernike polynomial and then sampled with different densities. The results for semianalytical ray tracing on the Zernike surface are shown for comparison, as they do not depend on the sampling density (number of facets). 


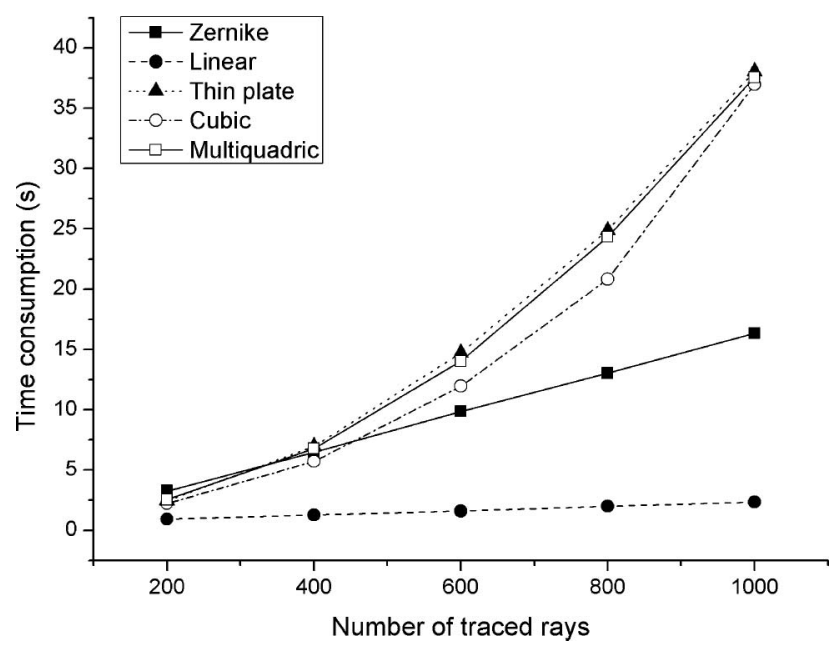

Fig. 8. Time consumption as a function of the number of traced rays for finite-element ray-tracing algorithm with various methods of data interpolation in comparison to the semianalytical procedure used with Zernike polynomial expansion.

be traced for the different interpolation methods used in the finite-element algorithm (sampling $20 \times$ 20 per $\mathrm{mm}^{-2}$ ) and for the Zernike semianalytical method. In general, finite-element algorithms are competitive for the number of rays up to 300 , above the typical number of rays used in visual optics applications [58]. For a linear interpolation, the time consumption is significantly lower due to relatively small effort in finding the normals to the surface at each ray intersection with the surface.

\section{Conclusions}

The algorithm presented, based on a Delaunayreconstructed surface, allows performing easily a ray tracing on a regular or random grid of points from either analytical, noisy, or free-form surfaces. We have shown that the ray tracing through analytical surfaces is accurate even for reasonably low density samplings (when compared to analytical results provided by optical analysis software). However, as in many other finite-element methods, denser sampling improves accuracy further when compared to the results obtained with the use of analytical methods.

The algorithm is able to determine the points of intersection and normals on noisy surfaces. The accuracy depends on the surface roughness, and decreases with increasing height $\mathrm{rms}$ and decreasing correlation length.

In principle, the Delaunay decomposition method is very flexible, allowing us to trace rays through any optical surface given by discrete data points. Unlike with modal surface fitting (such as in Zernike polynomials), surfaces are not necessarily smoothed. Together with well-described algorithms for tracing rays through gradient index structures [1-4], it can serve as a powerful tool for optical computations. The only limitation is the memory usage of the processing unit and the computational time, which might be very large for tracing many rays through densely sampled surfaces. We have shown that all methods tend to converge for densities above $40 \times 40$ samples. When the Delaunay decomposition is combined with a thin plate or cubic interpolation, the results from ray tracing are similar to those when a Zernike fitting and semianalytical ray tracing of the surface is used, for densities as low as $7 \times 7$ samples. The advantages of the Delaunay decomposition are its relative simplicity, higher speed, and increased efficiency in estimating the surface normals.

The presented method can be used to test the influence of the quality of topographical surface reconstruction on the optical performance, as well as the influence of the noise or high-frequency irregularities of the realistic surface on the performance of the optical elements; or the reversed question (i.e., what is the influence of the smoothing of the raw topography data of the real surfaces on their optical performance). Applications of this algorithm include the estimation of the aberrations produced by realistic surfaces and the application of the optical distortion correcting algorithms.

\section{References and Notes}

1. D. T. Moore, "Ray tracing in tilted, decentered, displaced gradient-index optical systems," J. Opt. Soc. Am. 66, 789-795 (1976).

2. A. Sharma, D. V. Kumar, and A. K. Ghatak, "Tracing rays through graded-index media: a new method," Appl. Opt. 21, 984-987 (1982)

3. E. W. Marchand, "Rapid ray tracing in radial gradients," Appl. Opt. 27, 465-467 (1988).

4. R. A. Egorchenkov and Y. A. Kravtsov, "Complex ray-tracing algorithms with application to optical problems," J. Opt. Soc. Am. A 18, 650-656 (2001).

5. O. N. Stavroudis, "Ray-tracing formulas for uniaxial crystals," J. Opt. Soc. Am. 52, 187-191 (1962).

6. Q. T. Liang and X. D. Zheng, "Ray-tracing calculations for uniaxial optical components with curved surfaces," Appl. Opt. 30, 4521-4525 (1991).

7. G. Beyerle and I. S. McDermid, "Ray-tracing formulas for refraction and internal reflection in uniaxial crystals," Appl. Opt. 37, 7947-7953 (1998).

8. M. Izdebski, "Ray and wave tracing in uniaxial crystals perturbed by an external field," Appl. Opt. 47, 2729-2738 (2008).

9. X. Tian, G. Lai, and T. Yatagai, "Characterization of asymmetric optical waveguides by ray tracing," J. Opt. Soc. Am. A 6, 1538-1543 (1989).

10. J. S. Witkowski and A. Grobelny, "Ray tracing method in a 3D analysis of fiber-optic elements," Opt. Appl. 38, 281-294 (2008).

11. W. A. Allen and J. R. Snyder, "Ray tracing through uncentered and aspheric surfaces," J. Opt. Soc. Am. 42, 243-249 (1952).

12. M. Herzberger, "Automatic ray tracing," J. Opt. Soc. Am. 47, 736-739 (1957).

13. G. H. Spencer and M. V. R. K. Murty, "General ray-tracing procedure," J. Opt. Soc. Am. 52, 672-678 (1962).

14. A. Sharma and A. K. Ghatak, "Ray tracing in gradient-index lenses: computation of ray-surface intersection," Appl. Opt. 25, 3409-3412 (1986).

15. R. A. Applegate, H. C. Howland, J. Buettner, A. J. Cottinghan, Jr., R. P. Sharp, and R. W. Yee, "Corneal aberrations before and after radial keratotomy (RK) calculated from videokeratometric measurements," in Vision Science and Its Applications, Vol. 2 of 1994 OSA Technical Digest Series (Optical Society of America, 1994), pp. 58-61. 
16. H. C. Howland, J. Buettner, and R. A. Applegate, "Computation of the shapes of normal corneas and their monochromatic aberrations from videokeratometric measurements," in Vision Science and Its Applications, Vol. 2 of 1994 OSA Technical Digest Series (Optical Society of America, 1994), pp. 54-57.

17. P. Artal and A. Guirao, "Contributions of the cornea and the lens to the aberrations of the human eye," Opt. Lett. 23, 1713-1715 (1998).

18. A. Guirao and P. Artal, "Corneal wave aberration from videokeratography: accuracy and limitations of the procedure," J. Opt. Soc. Am. A 17, 955-965 (2000).

19. A. Guirao, M. Redondo, and P. Artal, "Optical aberrations of the human cornea as a function of age," J. Opt. Soc. Am. A 17, 1697-1702 (2000).

20. S. Barbero, S. Marcos, and J. Merayo-Lloves, "Corneal and total optical aberrations in a unilateral aphakic patient," J. Cataract Refract. Surg. 28, 1594-1600 (2002).

21. L. A. Carvalho, "Computer algorithm for simulation of the human optical system and contribution of the cornea to the optical imperfections of the eye," Revista de Física Aplicada e Instrumentação 16, 7-17 (2003).

22. J. C. He, J. Gwiazda, F. Thorn, and R. Held, "Wave-front aberrations in the anterior corneal surface and the whole eye," J. Opt. Soc. Am. A 20, 1155-1163 (2003).

23. R. Navarro, L. González, and J. L. Hernández-Matamoros, "On the prediction of optical aberrations by personalized eye models," Optom. Vis. Sci. 83, 371-381 (2006).

24. P. Rosales and S. Marcos, "Customized computer models of eyes with intraocular lenses," Opt. Express 15, 2204-2218 (2007).

25. B. Delaunay, "Sur la sphère vide," Izvestia Akademii Nauk SSSR, Otdelenie Matematicheskikh i Estestvennykh Nauk 7, 793-800 (1934).

26. L. Guibas, D. Knuth, and M. Sharir, "Randomized incremental construction of Delaunay and Voronoi diagrams," Algorithmica 7, 381-413 (1992).

27. C. Touma and C. Gotsman, "Triangle mesh compression," in Proceedings of Graphics Interface '98 26-34 (Canadian Information Processing Society, 1998).

28. J. Kohout, J. I. Kolingerová, and J. Žára, "Parallel Delaunay triangulation in $E^{2}$ and $E^{3}$ for computers with shared memory," Parallel Comput. 31, 491-522 (2005).

29. D. S. Kang, Y. J. Kim, and B. S. Shin, "Efficient large-scale terrain rendering method for real-world game simulation," in Technologies for E-Learning and Digital Entertainment: First International Conference, Edutainment 2006 (Springer, 2006), pp. 597-605.

30. Zemax Optical Design Program User's Manual (Zemax Development Corporation, 2007).

31. S. Ortiz, D. Siedlecki, L. Remon, and S. Marcos, "Threedimensional optical distortion correction for quantitative anterior segment OCT,” Invest. Ophthalmol. Vis. Sci. 50, 5796 (2009), abstract.

32. A. Podoleanu, I. Charalambous, L. Plesea, A. Dogariu, and R. Rosen, "Correction of distortions in optical coherence tomography imaging of the eye," Phys. Med. Biol. 49, 1277-1294 (2004).

33. V. Westphal, A. M. Rollins, S. Radhakrishnan, and J. A. Izatt, "Correction of geometric and refractive image distortions in optical coherence tomography applying Fermat's principle," Opt. Express 10, 397-404 (2002).

34. G. Strang and G. Fix, An Analysis of the Finite Element Method (Prentice-Hall, 1973).

35. P. L. George and H. Borouchaki, Delaunay Triangulation and Meshing (Editions Hermes, 1998).

36. R. Vanselow, "About Delaunay triangulations and discrete maximum principles for the linear conforming FEM applied to the Poisson equation," Appl. Math. 46, 13-28 (2001).
37. N. Calvo, S. R. Idelsohn, and E. Onate, "The extended Delaunay tessellation," Eng. Computat. 20, 583-600 (2003).

38. I. Babuska, U. Banerjee, and J. E. Osborn, "Generalized finite element methods: main ideas, results, and perspective," Int. J. Computat. Methods 1, 67-103 (2004).

39. H. S. M. Coxeter, "Barycentric Coordinates," in Introduction to Geometry, H. S. M. Coxeter, ed. (Wiley, 1969), pp. 216-221.

40. It may happen that the point $P$ is located on the edge of the triangle (when either $u$ or $v$ from Eq. (6) is equal to 0 , or $u+v=1$ ), or even in the vertex (when both $u$ and $v$ are equal to 0 or either $u$ or $v$ is equal to 1 ), which means that it is common to two or more of the triangles that have a common edge (vertex), but the vectors normal to these triangles are different. In this situation, it is convenient to consider the mean value of the normal vector coordinates for further ray tracing calculations.

41. H. Wendland, Scattered Data Approximation (Cambridge U. Press, 2004).

42. F. L. Bookstein, "Principal warps: thin plate splines and the decomposition of deformations," IEEE Trans. Pattern Anal. Machine Intell. 11, 567-585 (1989).

43. J. Allison, "Multiquadric radial basis functions for representing multidimensional high energy physics data," Comput. Phys. Commun. 77, 377-395 (1993).

44. G. R. Liu, Mesh Free Methods: Moving Beyond the Finite Element Method (CRC Press, 2002).

45. It should be noted that in the original Sharma formula in [2], a square term of $v$ is missing. This has been corrected on Eq. (10).

46. J. Schwiegerling, J. E. Greivenkamp, and J. M. Miller, "Representation of videokeratoscopic height data with Zernike polynomials," J. Opt. Soc. Am. A 12, 2105-2113 (1995).

47. S. Barbero, S. Marcos, J. Merayo-Lloves, and E. MorenoBarriuso, "Validation of the calculation of corneal aberrations from videokeratography: a test on keratoconus eyes," J. Refract. Surg. 18, 263-270 (2002).

48. D. Malacara, Optical Shop Testing (Wiley, 1978).

49. L. Tsang, J. A. Kong, K.-H. Ding, and C. O. Ao, "Random rough surface simulations," in Scattering of Electromagnetic Waves: Numerical Simulations, L. Tsang, J. A. Kong, K.-H. Ding, and C. O. Ao, eds. (Wiley, 2001), pp. 124-132.

50. P. Beckmann, "Scattering of light by rough surfaces," in Progress in Optics, E. Wolf, ed. (North-Holland, 1967), pp. 55-69.

51 . W. N. Charman, "The eye in focus: accommodation and presbyopia," Clin. Exp. Optom. 91, 207-225 (2008).

52. D. J. Meister and S. W. Fisher, "Progress in the spectacle correction of presbyopia. Part 2: Modern progressive lens technologies," Clin. Exp. Optom. 91, 251-264 (2008).

53. M. K. Smolek and S. D. Klyce, "Is keratoconus a true ectasia? An evaluation of corneal surface area," Arch. Ophthalmol. 118, 1179-1186 (2000).

54. W. A. Douthwaite, "Application of linear regression to videokeratoscope data for tilted surfaces," Ophthal. Physiol. Opt. 22, 46-54 (2002).

55. V. A. D. P. Sicam, J. Coppens, T. J. T. P. van den Berg, and R. G. L. van der Heijde, "Corneal surface reconstruction algorithm that uses Zernike polynomial representation," J. Opt. Soc. Am. A 21, 1300-1306 (2004).

56. J. Turuwhenua, "Corneal surface reconstruction algorithm using Zernike polynomial representation: improvements," J. Opt. Soc. Am. A 24, 1551-1561 (2007).

57. D. R. Iskander, M. J. Collins, and B. Davis, "Optimal modeling of corneal surfaces with Zernike polynomials," IEEE Trans. Biomed. Eng. 48, 87-95 (2001).

58. L. Llorente, S. Marcos, C. Dorronsoro, and S. A. Burns, "Effect of sampling on real ocular aberration measurements," J. Opt. Soc. Am. A 24, 2783-2796 (2007). 